Meta

Journal des traducteurs

Translators' Journal

\title{
Pour une définition de la lexicologie
}

\section{Alain Rey}

Volume 18, numéro 1-2, mars 1973

Actes du deuxième colloque international de linguistique et de traduction. Montréal, 4-7 octobre 1972

URI : https://id.erudit.org/iderudit/003945ar

DOI : https://doi.org/10.7202/003945ar

Aller au sommaire du numéro

Éditeur(s)

Les Presses de l'Université de Montréal

ISSN

0026-0452 (imprimé)

1492-1421 (numérique)

Découvrir la revue

Citer cet article

Rey, A. (1973). Pour une définition de la lexicologie. Meta, 18(1-2), 9-18.

https://doi.org/10.7202/003945ar d'utilisation que vous pouvez consulter en ligne.

https://apropos.erudit.org/fr/usagers/politique-dutilisation/ 


\section{Pour une définition de la lexicologie}

\section{STATUTS CONCRETS DE LA LEXICOLOGIE}

La lexicologie, discipline constituée et admise dans la tradition linguistique européenne, peut recevoir plusieurs statuts. Ainsi, dans le monde scientifique anglosaxon, elle n'est guère reconnue que comme un objet interdisciplinaire dont les techniques, assez éloignées de celles de la "linguistique », relèvent de l'anthropologie, de l'ethnologie (cf. les travaux de Conklin, Berlin, etc.).

Pourtant, une évolution s'amorce, et, à côté de la lexicographie, à laquelle la plupart des linguistes américains réduisent la lexicologie, on cherche à définir une théorie lexicologique.

De fait, la lexicographie ne s'identifie pas, pour nous, avec la lexicologie : ces deux disciplines sont plutôt en relation de complémentarité. En effet, la lexicographie : $a$ ) déborde par son objet le domaine lexical, $b$ ) constitue un type de discours sur la langue qui a ses règles propres, relevant de la théorie du discours, c) n'est que la plus continue des pratiques linguistiques portant essentiellement sur la signification.

1.1. Or, la réflexion sur le langage, en Occident, a d'abord été une réflexion sur le signe, et notamment sur un signe linguistique privilégié, le signe lexical (témoins Aristote, les stoïciens, la sémantique médiévale après Augustin et jusqu'aux modistes et une grande partie de la philosophie classique du signe).

L'importance relative de cette sémantique lexicale et de la philosophie du nom a diminué avec l'apparition d'une linguistique des formes fonctionnelles (depuis Rask et Bopp). Pourtant, elle est restée vivante, non seulement en philosophie du langage, mais à l'intérieur de la science linguistique, par les pratiques de la philologie, et elle a ressurgi avec le passage du comparatisme à l'historicisme plus exigeant des Junggrammatiker, et surtout avec la naissance d'une sémantique explicite des unités-signes, d'abord diachronique (Reisig, Bréal), puis fonctionnelle et structurale (après Saussure).

1.2. Pour des raisons pratiques (sociales), la description du lexique a joué un rôle prépondérant non seulement en lexicographie, mais dans l'enseignement des langues, dans la traduction et en général dans toutes les pratiques langagières conscientes et plus ou moins normalisantes : élaboration des terminologies (ex. : Lavoisier), interventions sur le langage, pédagogie, etc. 
La maîtrise intuitive des régularités du système étant toujours plus complète que celle des irrégularités de la norme, il allait de soi que l'activité traductrice ou pédagogique devait s'occuper (pratiquement et explicitement) du lexique, la grammaire étant pour elle un présupposé implicite. De même, les variations de la norme sont plus grandes et plus rapides en matière lexicale, et l'action normative s'exerce plus fréquemment dans ce domaine (d'où l'intérêt pour le néologisme, l'emprunt, les structures terminologiques).

1.3. En outre, les moyens techniques récents mis à la disposition du linguiste, mécanographie et électronique, s'articulent mieux avec les problèmes de stockage, de classement, de repérage d'unités nombreuses (morphèmes ou mots) qu'avec ceux de l'application de règles peu nombreuses mais organisées de manière si complexe qu'on ne peut pas encore fournir aux mécanismes un programme approprié (cf. les problèmes insolubles que pose la grammaire, notamment la syntaxe sémantique, à la traduction automatique).

1.4. À ces considérations historiques et pragmatiques, il faut ajouter une remarque théorique. Le mouvement de scientifisation des connaissances anthropologiques et l'approfondissement de 1a «philosophie du sens » conduisent à donner une importance accrue aux problèmes de la principale unitê-signe de la sémiotique et de l'anthropologie, qui est le mot (l'unité-signe centrale en linguistique étant soit le morphème, soit certains syntagmes, soit la phrase type (sentence).

$\AA$ ceci répond un fait concret : en même temps que la linguistique descriptive distributionnelle, suivie par le générativisme, s'est dégagée de l'ethnologie (linguistique à tendance sociologique depuis Boas, anthropologue, jusqu'à Sapir, Whorf, puis à Weinreich et à la socio-linguistique), de même, de la philologie comparée et historique est sortie une linguistique socio-historique. La scientifisation de la sémantique, notamment en Europe, a donné lieu à des tentatives de linguistique globale incluant toutes les questions de la sémantique lexicale (ex. : Wartburg, les sémanticiens français, les structuralistes de l'école allemande, etc.).

Toutes ces tendances, nourries par les réflexions des principaux linguistes européens (de Saussure et Bally à Benveniste ou Hjelmslev et ses disciples) et d'Américains comme Pike accordent une grande importance à la structuration de la «substance du contenu » par la forme et rencontrent au centre de leurs préoccupations le lexique et le mot.

\section{L'ARTICULATION DU LEXIQUE DANS LE SYSTÈME DE LA LANGUE}

Elle pose un problème majeur, et a pu conduire des linguistes pourtant animés par un esprit sociologique à contester l'existence même de la lexicologie.

2.1. L'exemple le plus célèbre est sans doute celui de Saussure, qui après Whitney, cherche à rendre compte des conditions spécifiques de la fonctionnalité du système linguistique. Grâce aux concepts de «langue » et de «synchronie », on le sait, Saussure est parvenu à élaborer une linguistique qu'il définit comme la science des «systèmes de moyens d'expression ». Le Cours de linguistique générale bat en brèche les divisions traditionnelles entre morphologie, syntaxe et lexicologie. 
La morphologie est résorbée : " elle ne peut constituer une discipline distincte de la syntaxe» (II, chap. 7). Quant à la lexicologie, son objet est le « mot». Or, si l'on pense le «mot» en termes de rapports fonctionnels, sa spécificité s'effondre. Ainsi, l'opposition fonctionnelle perfectif/imperfectif peut être rendue en russe, note Saussure, par une opposition de signifiants de nature morphologique (donc grammaticale) ou de nature lexicale (sprosit'/sprašivat' c. skazat'/govorit' : \& demander» et «dire»). Le rôle des prépositions (fr. le royaume des cieux) peut être assumé par la composition d'éléments lexicaux (all. Himmelreich). II semble même que Saussure se laisse entraîner sur cette voie à utiliser d'assez mauvais arguments, comme celui de la réalisation d'une même opposition sémantique par deux syntagmes nominaux comportant une préposition (bois de chauffage/bois de construction) ou par deux lexèmes (russe drova/lês), la première étant «grammaticale». A vrai dire, je ne vois pas en quoi la présence de la préposition de dans les deux syntagmes français diminue le caractère « lexicologique $\gg$ de l'opposition chauffage/construction, ni pourquoi la neutralisation de l'opposition en contexte (bois), fait de polysémie, entrerait dans la «syntaxe».

Dire, comme le fait le C.L.G., qu' « au point de vue de la fonction », le fait lexicologique peut se confondre avec le fait syntaxique, revient simplement à admettre que les éléments du lexique sont tenus de fonctionner syntactiquement, c'est-à-dire, dans les termes de Saussure, que considérer la fonctionnalité consiste à se placer en linguistique «syntagmatique ». Plus intéressant est le fait suivant : constater que «tout mot qui n'est pas une unité simple et irréductible ne se distingue pas essentiellement d'un membre de phrase, d'un fait de syntaxe », revient à assimiler la morphologie à la syntaxe et à réduire le fait lexical au morphème.

2.2. En fait, toute attitude descriptive et fonctionnaliste conduit, par une démarche analytique et inductive, à extraire de la syntagmatique (qui est une neutralisation méthodologique des observables du discours) des unités fonctionnelles. Ainsi, Martinet préfère considérer «pour la compréhension des fondements de la structure linguistique $\gg$ (Éléments de linguistique générale, p. 115) une unité différente du mot : le «syntagme autonome ». C'est à juste titre, dans son optique, puisque la compréhension des fondements de la structure dérive chez lui d'une analyse inductive des phénomènes récursifs du discours. Dans cette optique, le «mot» est un type particulier de syntagme autonome (l'auteur compare d'une manière très saussurienne lat. homini à esp. para el hombre, fr. pour l'homme, le premier n'étant caractérisé, dit-il, que par des monèmes plus inséparables).

Le concept de «mot», chez Martinet, comme chez Harris, etc., est subordonné aux concepts fonctionnels inductifs de «morphème », ou «monème » et de «syntagme ». Quant au «lexique », il ne peut être qu'un résidu gênant, et doit s'identifier à un stock de morphèmes.

2.3. Il est intéressant de confronter, en ce qui concerne l'attitude envers les problèmes lexicaux, la linguistique post-saussurienne et post-bloomfieldienne avec la linguistique générative (sous sa forme chomskienne «classique», celle de Aspects of the Theory of Syntax). Là encore, la simplicité nécessaire du modèle incite à considérer le lexique comme une liste de morphèmes soumis à des règles. L'absence de régularité complète — ou l'irrégularité partielle — des phénomènes de la mor- 
phologie conduirait, au contraire, à introduire directement dans le lexique des éléments supérieurs au morphème (mots « dérivés», «composés», etc.). Mais, comme le note Chomsky (Aspects, p. 252 de la trad. française) « ce serait là une conclusion très regrettable, puisqu'il est manifestement important, du point de vue de l'interprétation sémantique et phonologique, qu'une structure interne soit représentée par ces mots ». C'est tout le problème des limites d'application des règles morpho-sémantiques et de la démotivation des éléments qui est reflété dans les hésitations théoriques, d'une extrême honnêteté intellectuelle, qui sont formulées dans les derniers chapitres de l'ouvrage classique de Chomsky. Ces hésitations illustrent parfaitement la difficulté à définir un concept rigoureux de « lexique », notamment dans une théorie hypothético-déductive recherchant l'explicitation totale.

\section{DÉFINITIONS POSSIBLES DU LEXIQUE}

Il $\mathrm{y}$ a trois manières principales, d'ailleurs bien connues, d'envisager le lexique selon les besoins méthodologiques et heuristiques.

3.1. Le lexique comme ensemble des morphèmes (ou monèmes) d'une langue. Cette définition est adoptée par la plupart des linguistes fonctionnalistes et distributionnalistes. Pour la linguistique générative, ce concept est une commodité et une tentation, partiellement repoussée. Il se heurte à deux types de difficultés : a) Quelles que soient les règles de la morpho-syntaxe, leur application à un tel «lexique » produit un ensemble de signes minimaux libres, supérieurs à ce que l'on peut observer dans le fonctionnement de la langue. On obtient alors un système linguistique trop puissant, correspondant au schéma hjelmslevien, et la description saute au moins un niveau d'intégration rendant compte du passage du morphème aux unités de la communication (la norme de Hjelmslev) ; b) L'opposition entre paradigmes ouverts et fermés (ou indéterminés et prédéterminés, quant au choix du locuteur) confère à ce concept du lexique un caractère hybride. Ainsi, les morphèmes grammaticaux et eux seuls peuvent être sélectionnés de manière univoque au moyen d'un ensemble fermé de traits fonctionnels.

3.2. Le lexique peut être considéré comme l'ensemble des «mots», c'est-à-dire comprendra la plupart des morphèmes libres envisagés comme intégrants du syntagme (phrase) et indirectement de la phrase (sentence), ainsi que toutes les unités formées de plusieurs morphèmes ayant ce même caractère d'intégrant. On se trouve done renvoyé aux difficultés de la définition du «mot » et de l' « intégrant » (au sens de Benveniste), et au concept d'《unité codée», qui concerne la fonctionnalité informationnelle. Cette définition du lexique est tributaire de la notion de «partie du discours» (que met en cause la sémantique d'inspiration logicienne, cf. dans ce colloque la communication de M. Querido), et l'unité du «lexique » y est encore menacée par la dichotomie entre «mots grammaticaux» et «mots lexicaux », les premiers étant fonctionnellement identiques à des morphèmes liés (cf. les remarques de Saussure ci-dessus, et les comparaisons inévitables entre les flexions du latin, etc., et les prépositions du français, ou entre l'article-mot du français et l'article-morphème postposé du roumain, etc.). 
Il convient de noter que cette définition du lexique est pragmatiquement essentielle : c'est celle des dictionnaires de langue, qui commencent d'ailleurs à la contester en traitant certains morphèmes liés (préfixes, suffixes).

3.3. Pour peu que l'on donne la priorité aux considérations fonctionnelles interou intralinguistiques, on est conduit à définir le lexique comme un ensemble indéterminé mais fini d'éléments, d'unités ou d' «entrées » (dans les théories génératives) en opposition aux éléments réalisant directement des fonctions « grammaticales » (telles que la fonction « déterminant», " auxiliaire», etc.). Cet ensemble peut correspondre aux morphèmes lexicaux et à leurs productions codées (chez Martinet, par exemple) ou à l'ensemble des «idiomes » (mots lexicaux et locutions).

Dans la pratique, le lexique est souvent considéré comme l'ensemble des mots à fonction non "grammaticale 》, i.e. des noms, des verbes, des adjectifs et de la plupart des adverbes; il exclut alors les morphèmes liés (en français, des suffixes comme -ment, des préfixes comme re-) et les mots dits "grammaticaux», la frontière étant d'ailleurs très indistincte.

3.4. On est donc en présence d'une définition trop large (l'ensemble des morphèmes) et de plusieurs définitions basées sur des concepts mal délimités (celui de «mot », d' « idiome », ou celui de «fonction grammaticale », opposée à « fonction lexicale »). Le concept de «mot», d'origine graphique, et par-delà l'écriture, d'origine intuitive (donc sémantique) est d'ailleurs dominé par celui d'unité codée (concept informationnel). A noter que la frontière entre suite de morphèmes entrant comme telle dans le code, et suite libre, est essentielle à la définition d'un lexique (cf. les remarques de Hockett, Coseriu, Greimas et ma communication au congrès des romanistes de Québec en 1971).

\section{VERS UNE DÉFINITION DE LA «LEXICALITÉ "}

La seule définition possible de la lexicalité des unités (ou, si l'on préfère, la seule définition du «mot $\gg$ ) est de nature sémiotique (au sens hjelmslevien). En effet, alors que la syntaxe traite toujours de la forme (forme de l'expression et forme du contenu), la lexicologie doit traiter de la forme dans ses rapports avec la substance du contenu.

Son objet ne peut être constitué que par l'importance relative de ces rapports, dans ce qu'ils ont de descriptible et d'analysable. Le morphème et le mot " grammaticaux » sont sémantiquement déterminés par leur fonction, qui reflète la structure immanente de la langue. Leur sens est entièrement investi par le code syntactique ou par la situation de communication (cas des pronoms personnels, des adverbes de lieu et de temps). Au contraire, les signifiés des mots «lexicaux» sont (relativement) indépendants du système abstrait de la langue (sa grammaire) et sont sémantiquement analysables (au moins partiellement) hors contexte (d'où la possibilité de paraphrase synonymique : définition de dictionnaire).

En général, plus les unités lexicales sont indépendantes de la fonctionnalité grammaticale et moins elles sont fréquentes (en discours), plus elles sont nom- 
breuses en langue (cf. les énormes paradigmes de mots rares des terminologies scientifiques taxinomiques, en zoologie, botanique, etc.). On peut proposer une échelle correspondant à un passage progressif de la sémantique fonctionnelle syntactique à la sémantique analytique intentionnelle, enfin à la seule possibilité d'une sémantique extensionnelle, référentielle (pour l'unité en langue, toute unité analysable correspondant évidemment à une possibilité référentielle en discours). Cette échelle serait d'ailleurs un continuum où les seuils dépendent des nécessités méthodologiques; elle irait des mots «grammaticaux » aux adverbes, aux verbes, aux adjectifs et enfin aux noms, puis aux noms propres. Le domaine central de la lexicologie correspondrait dans cette optique au nom (commun) et aux aspects extra-fonctionnels de l'adjectif et du verbe, liés au nom par les règles morpho-sémantiques de transformation (nominalisations, adjectivations, etc.). Ce domaine est celui même des rapports entre forme et substance du contenu, celui de la compatibilité entre structures linguistiques et structures cognitives.

D'un côté, celui de la grammaire, les structures linguistiques sont immanentes et les structures cognitives en dépendent. S'il y a mise en relation du système linguistique et des données anthropologiques (et cette mise en relation est évidemment indispensable), elle ne peut être qu'ultra-profonde (structures logiques et psychologiques universelles).

De l'autre côté, celui du domaine lexical, et notamment en son centre, dominé par les relations de désignation, de nomination (name), et par la conceptualisation, les structures linguistiques du signifié sont dépendantes de toute l'appréhension du monde par l'homme social. La «linguistique » pure est insuffisante (mais indispensable) pour en rendre compte et la contribution de sciences comme la psychologie et la psychanalyse, l'ethnologie et la sociologie, l'épistémologie, enfin l'histoire et les théories du discours (notamment la poétique), devient absolument nécessaire malgré d'évidentes difficultés méthodologiques pour conserver la cohérence d'un modèle.

\section{SYSTÉMATICITÉ DU LEXIQUE}

Le lexique est sans aucun doute l'irrégularité fondamentale, face aux régularités de la syntaxe (et de la phonologie). Mais cette formule de Bloomfield, explicitement reprise par Chomsky, et qui a ses racines dans la plus ancienne philosophie du langage (l'anomalia des Anciens) peut donner lieu à deux attitudes.

5.1. On peut tenter de dégager les limites de la régularité dans la langue, afin de dégager les limites mêmes de la théorie linguistique, qui ne peut être construite que dans le domaine des «lois». On l'a vu, si l' « on incorpore à la grammaire des règles [lexicales] très générales qui autorisent les cas non attestés aussi bien que les autres» (Chomsky, Aspects, p. 253), on obtient une description trop puissante et on néglige tous les aspects socio-sémantiques du langage; on décrit le schéma abstrait, mais non la norme. On peut aussi étendre «la théorie du lexique pour permettre une sorte de «calcul interne» au lieu de l'application simple de la règle lexicale générale » (ibid.), ce qui revient à assigner à la description une zone des régularités partielles et à délimiter une lexicologie lin- 
guistique «pure », menacée soit d'insuffisance par rapport aux observables, soit de devenir une liste interminable de sous-régularités de plus en plus fines (les listes d'exceptions des grammaires traditionnelles en donnent le déplorable exemple).

5.2. On peut aussi, comme on le fait dans toutes les sciences de l'homme, et notamment dans les sciences historiques, s'assigner les irrégularités apparentes comme objet de savoir, afin de les réduire. Ce qui peut se tenter, soit dans une optique descriptive et taxinomique, soit dans une optique «nomothétique 》(Piaget), c'est-à-dire en cherchant d'autres lois ou règles quand celles qu'a établies la linguistique font défaut; ce qui suppose un appareil d'hypothèses et une méthodologie de la vérification par observation. Il s'agira alors de régularités logiques, informationnelles, psychologiques et psychanalytiques, sociologiques, historiques, etc. Certes, un tel programme, trop ambitieux, couvre tous les domaines du langage et dépasse largement l'étude du système, et même celle de la norme ; on peut cependant penser qu'une science générale du langage en dépend, et que la lexicologie est l'un de ses principaux aspects.

La première démarche, descriptive, est indispensable. Les inventaires à quoi elle aboutit ne sont pas une fin en eux-mêmes, mais permettent déjà de dégager dans la masse des faits lexicaux des sous-systèmes tels que des structures synchroniques lexico-sémantiques (champs, inventaires des relations sémantiques du type antonymie, synonymie, hypo-hypéronymie, etc. ; cf. les travaux de Trier, Weisgerber, Coseriu, etc., la sémantique componantielle des ethno-linguistes, de Pottier ou des générativistes, comme Bierwisch), des structures diachroniques (la « sémantique $\gg$ de Stern, Ullmann, etc., les structures étymologiques étudiées par Guiraud...). Dans le domaine du discours, on connaît les travaux d'analyse du récit inspirés de Propp (Greimas et ses élèves, en France). Tous ses sous-systèmes présentent des relations descriptibles qui ne sont pas quelconques et leur étude ouvre la voie à la recherche de véritables lois.

La seconde démarche conduit à envisager, outre le domaine d'applicabilité des règles internes qui déterminent le système de la langue (et du point de vue de la productivité, la compétence), l'étude de régularités dans le domaine psychophysiologique de la réalisation (ou de la performance chomskyenne) et, entre ces deux zones, un domaine essentiellement socio-historique qui est celui de la norme objective.

Il existe de nombreuses applications grammaticales et phonologiques du concept de norme objective, mais il semble que la lexicologie soit son domaine privilégié, dans la mesure où il est celui de la variation par rapport aux structures fixes du schéma, du système abstrait.

L'irrégularité du lexique, reconnue à juste titre au niveau fondamental, cède la place à des régularités partielles au niveau socioculturel, le concept de langue s'affinant par celui de dialecte. Les faits de lexique, qui plongent dans la structure globale de la langue (morpho-syntaxe, phonologie de l'unité lexicale), sont inexplicables, comme tous les faits de norme, sans recours à l'étude des instances de l'énonciation, des conditions socioculturelles de la communication, des conditions 
(logico-sociales) de la conceptualisation, pour ne mentionner que quelques domaines essentiels. Là, sans doute, doit résider la causalité des variations de la norme objective à l'intérieur des potentialités du système que définissent et analysent les théories linguistiques pures, et notamment celles du lexique.

6. La situation ouverte, transdisciplinaire, de la lexicologie, a été trop bien reconnue, intuitivement, par ceux qui se réclament ouvertement d'une discipline autonome pour l'étude du lexique (cf. la Méthode en lexicologie de G. Matoré). Mais toute chance d'élaborer une lexicologie scientifique passe par l'exploitation maximale des possibilités d'explicitation et de formalisation, que seule la linguistique pure est (pour le moment) en mesure d'exploiter. Ceci posé, il faut admettre que cette exploitation maximale est encore insuffisante pour rendre compte des faits de lexique - et en général des faits de norme et des faits de réalisation - ce qu'illustre le caractère fictif du concept de «compétence lexicale», comparé à celui de « compétence syntaxique ».

La vocation transdisciplinaire de la lexicologie m'est apparue clairement lors d'une discussion récente sur l'œuvre de Bachelard. On cherchait à définir, difficilement, l'unité de travaux abordant à la fois l'épistémologie des sciences exactes, le surgissement de la pensée rationnelle et les racines de l'imaginaire. Pour moi, cette unité est dans la sémantique du signe lexical, qui rend compte des relations entre signifiés conceptualisés et connotations socioculturelles, jusqu'aux structures de l'inconscient collectif et de l'imagination poétique.

Science-carrefour des disciplines anthropologiques et domaine essentiel de l'activité pragmatique sur le langage, la lexicologie est dominée méthodologiquement par la linguistique et épistémologiquement par la sémiotique (pour éviter le terme dangereux de «philosophie du signe $»)$. Si l'on accepte les présupposés et les hypothèses de cette communication - beaucoup peuvent sans doute en être discutés - ceci devrait articuler la définition d'une science encore à naître.

Alain ReY

\section{QUESTIONS}

M. Provencher : J'ai été frappé par votre conclusion que la lexicologie serait au centre des sciences humaines. Ne s'agit-il pas là un peu de ce que certains appelleraient du nombrilisme?

Réponse : Vous pouvez mettre sur le compte d'un certain lyrisme qui n'est pas absolument hors de mise au début d'une réunion comme celle-ci, une conclusion qui dépassait peutêtre un peu, dans sa formulation, une attitude plus neutre et plus scientifique, mais je crois sincèrement que si la lexicologie n'est pas le centre des sciences humaines - je ne crois pas avoir dit exactement cela — elle se situe à un carrefour et à un lieu d'intersection de domaines qui sont ceux des diverses sciences humaines. Cela ne veut évidemment pas dire qu'il n'y ait pas d'autres lieux d'intersection et que, pour leur compte, le sociologue ou le psychanalyste ne puissent aussi revendiquer une situation centrale dans les sciences de l'homme. Seulement je crois que, dans la mesure où la formation de la pensée doit obligatoirement s'appuyer sur un langage, dans la mesure où un langage est un code, dans la mesure où un code est formé d'unités et de règles et dans la mesure où les unités 
dans le cas du code naturel des langues font les unités du lexique, il est impossible de dissocier aucun aspect de ce qui est étudié dans les sciences de l'homme de l'aspect lexicologique. Maintenant, bien entendu, ce qui a été dit pour la délimitation d'un domaine n'est pas valable pour la caractérisation d'une méthode. Je ne dis pas que les méthodes lexicologiques ne sont pas données en exemple aux autres sciences humaines, à Dieu ne plaise! Je crois au contraire que ce sont les méthodes qui existent et qui se développent dans les autres sciences humaines qui sont et qui doivent être acceptées, testées, vérifiées ou rejetées par la lexicologie.

$M$. Provencher : J'ai entendu, durant votre exposé, plusieurs, je dirais même de nombreux mots nouveaux, comme scientifisation, lexicalisation, etc. Je pense que ce sont des mots qui sont nécessaires. Puis-je vous demander, à titre de lexicologue, quels sont les critères qui nous permettent de lancer un mot nouveau quand on en sent le besoin ? Quels critères devons-nous observer?

Réponse : Je crois que c'est un critère d'économie, dans le cas présent je préfère employer "scientifisation" que stendance à la constitution d'une scientificité ", qui lui-même est un mot complexe et pas très joli, ou "tendance à la constitution d'une attitude scientifique ", si on veut un syntagme qui évite toute nouveauté. C'est une question d'économie d'information.

M. Coseriu : J'ai tout d'abord une petite observation historique à faire à propos de l'excellent rapport de $M$. Rey en ce qui concerne la fondation de la sémantique historique. M. Rey l'a dit et tout le monde le dit, hélas, Bréal a répandu la sémantique historique. Ceci n'est pas tout à fait exact; elle existait déjà sous le nom de sématologie; elle avait été fondée par Rashley déjà autour de 1830, et même avant Bréal, une sémantique historique aussi sous le nom de sématologie avait paru pour le roumain et en français ensuite. J'ai beaucoup apprécié dans le rapport de M. Rey ce qu'il a dit à propos de la définition du lexique. Je crois être d'accord entièrement avec lui en ce qui concerne les difficultés de définir le lexique, si l'on veut tenir compte en même temps de l'expression et du contenu. Je crois qu'il va dans la même direction que moi, c'est-à-dire qu'il considère aussi que la seule possibilité cohérente de définir un objet de la lexicologie, c'est de définir un signifié lexical. En effet, on peut distinguer quatre types de signifiés : un signifié instrumental, un signifié grammatical, un signifié catégoriel et un signifié lexical. Par exemple, dans un mot espagnol tel que blancos le pluriel, $s$, est un signifié instrumental, c'est le signifié pluralisateur. Le mot tout entier blancos, considéré dans une place où l'on a aussi verdes, mesas, hombres et ainsi de suite, a un signifié grammatical qu'on appelle pluriel. Verde, blanco et ainsi de suite, ont un signifié qui leur est commun qui est le signifié catégoriel et blanco, blancura par exemple, c'est-à-dire blanc substantif et blanc adjectif, ont un signifié lexical; alors il s'agit tout simplement de constater l'existence du signifié lexical, d'étudier le signifié lexical dans les formes où il se présente. Mais ces formes n'ont pas que des signifiés lexicaux, elles ont aussi des signifiés instrumentaux, des signifiés grammaticaux. Je crois que ma conclusion serait la même que celle de $M$. Rey dans ce cas, même si je l'exprime au moyen d'une formulation différente, c'est-àdire, en réalité l'objet de la lexicologie, c'est le nom mais dans un sens qui n'est pas celui de nom substantif. Il s'agit de condition de la désignation, c'est-à-dire du sens dans lequel les verbes, les adjectifs sont aussi des noms, chose qui a été bien vue du reste à une époque très ancienne, à savoir par Aristote dans le De interpretatione où il dit explicitement que le verbe, c'est aussi un nom. Il considérait les adjectifs, évidemment, aussi en tant que noms, c'est-à-dire qu'ils sont des noms dans le sens de la désignation de la réalité, dans le sens de signifié lexical.

Réponse : En ce qui concerne la priorité d'une description de la sémantique historique, je suis tout à fait d'accord avec vous, je connaissais Rashley, bien sûr, et je crois simplement que Bréal a une importance plus grande au point de vue de l'histoire de la linguistique, concrètement, parce qu'il a plus articulé sa définition de la sémantique sur une mutation de la linguistique qui correspond à celle des Junggrammatiker exactement au même moment, et qui a permis de passer d'une linguistique comparative, qui n'avait pas encore une exigence de définition d'historicité, à une linguistique plus strictement historique. Quant à votre deuxième remarque qui est beaucoup plus importante et générale, je crois en effet qu'il s'agit sim- 
plement d'une différence, disons de formulation, et d'une différence de conceptualisation aussi, parce que vos signifiés grammaticaux et instrumentaux correspondent incontestablement à ce que j'ai désigné sous le nom de fonctionnalité. Le signifié lexical est tout entier dans ma lexicalité, mais votre signifié catégoriel me pose des problèmes, parce que pour moi il est déjà partiellement lexical. Autrement, c'est une différence de découpage conceptuel et l'esprit général est bien celui que vous avez défini en ce qui concerne l'appréhension du mot en tant que possibilité de nomination du monde tel qu'Aristote l'avait déjà défini. C'est vraiment central, c'est tout à fait le milieu du problème. 